creosote, or with 5 to 10 per cent. of ichthyol, or tincture of camphor, is always a safe and generally beneficial dressing if well and thoroughly applied. But to be of service it should be kept thickly applied, spread on a lint in most places, and bound on firmly. In the acutely inflamed and especially in the erythe. matous forms of the eruption, there is nothing better than the well known calamine and zinc lotion, freely sopped on many times in the day. Ichthyol in watery solution, 10 to 20 per cent, freely bathed on the part, often gives great relief, or if the skin is too dry it may be used in oil in the same strength.

On more chronic patches the permanganate of potas. sium, 2 per cent. solution in water, painted over the part will sometimes arrest the itching very well; it may either be used alone, or the calamine and zinc lotion may be sopped on after the surface has dried, or an ointment may be applied if the skin is at all hard.

In the erythematous eczema of the face a tannin ointment, 1.95 to 3.9 grams, to the 31.2 grams, with 2 per cent. of carbolic acid is effective; a mixture of camphor, 1.95 grams to 31.2 grams of zinc ointment also forms a good antipruritic. In still more chronic states the tar and zinc ointment, as recommended by the present writer many years ago, when thickly spread on lint and bound on, will often also serve admirably to control the itching.

The use of very hot water for a brief application, followed by an appropriate ointment, should never be forgotten. In old cases of eczema of the scrotum the effect of this treatment is sometimes very remarkable. Menthol will often prove a valuable addition to ointments, in the strength of 2 to 4 per cent., with about half as much carbolic acid, which latter serves both to heighten the antipruritic effect and also to overcome some of the chilly sensation caused by the menthol.

In bringing this brief and rather imperfect study of neurotic eczema to a close, I wish to again emphasize what was intimated early in my remarks; namely, that I do not regard eczema as a local condition or disease of the skin, but that it has constitutional and general relations which should always be considered and studied. The neurotic relations of eczema are especially interesting and important because of the excellent results which follow a careful and correct appreciation, interpretation and treatment of them; whereas, a neglect of this aspect of these cases will lead not only to their chronicity and rebelliousness, but also to an injury to the patient and further nerve exhaustion, whose ultimate result may be most deplorable.

4 East 37th St.

DISCUSSION.

Dr. SCHAMBERG-I will narrate briefly the history of a case of neurotic eczema. A young woman suffered great mental strain nursing her husband through a protracted illness. His death was a shock which prostrated her entirely. She thereafter suffered from a profound neurastbenia which lasted for a considerable period of time. She subsequently became restored to health by taking a rest cure. This woman consulted me for a recurring papular eczema of the hands and forearms. A peculiarity of this case was the extremely rapid evolution of the lesions. During an attack of mental excitement the papules would spring up almost under the eye of the observer. They were closely aggregated in patches and were intensely itchy. They disappeared in as short a time as they appeared. A noteworthy fact in this case is that a hysterical daughter was also the victim of eczema. There would thus seem to be an apparent tendency to heredity in this case.

Dr. FLEISCHNER - I saw a case of a young man of phlegmatic temperament, all depressing nervous elements entering into the disease of no apparent import. No treatment constitutional or local had any effect. It was found out indirectly that he was engaged to be married, and on being advised to ignore his disease altogether and fix a date for the wedding, his eczema abated without further treatment from the day of this advice being given. It is held that whenever the cause can be removed that alone will overcome the disease and unless that is done no amount of local or constitutional treatment will be of any but the most meager effect.

Dr. RAYogLI-I had an experience. with one patient who, whenever he smoked a cigar had an attack of eczema scroti.

Dr. BULKLEY - Tobacco will sometimes occasion attacks of eczema and I have repeatedly known it to be the cause of a relapse.

\section{MYCOSIS FUNGOIDES; WITH REFERENCE TO A CASE.}

Presented in the Section on Cutaneous Medicine and Surgery, at the Forty-eighth Annual Meeting of the American Medical Associaton held at Philadelphia, Pa., June 1-4, 189 .

BY JOHN V. SHOEMAKER, M.D., LL.D.

PHILADELPHIA, PA.

A married woman, 60 years of age, who, upward of a year ago, was for some time under my care, gave the following history: The disease from which she suffered began four years and three months prior to her first visit. The first manifestation occurred in the form of red patches situated upon the back. They were accompanied by intense itching. They were rather bright in color. From the back the lesions gradually spread to the breast, abdomen, neck and limbs, attacking even the soles of the feet. Some, after a certain degree of development, healed. According to her statement, the skin had been more seriously affected than it was at the date of the examination. Itching had throughout been a prominent symptom. The lesions were also the seat of considerable pain. They often bleod when rubbed or scratched.

The patient's appetite was poor, she was habitually constipated, and had lost flesh. She suffered also from insomnia. No case of inveterate skin disease had ever occurred in any member of her family. The patient is ignorant of any cause of this disease. When examined, Dec. 20, 1895, the greater part of the woman's body was found to be covered with patches and tumors of various sizes and stages of development. The face was comparatively free. A red, infiltrated patch was present upon the right upper eyelid. The thyroid gland was decidedly enlarged, more particularly upon the right side. The goiter had existed for many years before the appearance of the skin disease. Upon the skin covering the gland were several fungous excrescences. Numerous similar outgrowths were situated upon both sides of the neck. The upper part of the chest, the mammary glands, abdomen, and axillæ were extensively diseased. It is scarcely possible to describe the lesions in detail for the reason that their numbers, size and proximity left but little of the surface unaffected. The lesions consisted of patches and tumors. The former were of various sizes and shapes, of rather a bright red color, and more or less elevated. The latter were prominent outgrowths, some bright and others rather clark red, excoriated and often bleeding. They were broad at the base. A growth about four inches in average diameter, the base of which was rather oval than circular in outline, was seated upon the upper part of the left breast immediately in front of the axilla. Upon the right breast, in a nearly corresponding situation, was a long, oval, slightly elevated, bleeding patch. The anterior and posterior surfaces of the arms and forearms were studded with patches. Upon the back the lesions, though abundant, were not as 
numerous as upon the front of the body. Behind the right axilla there was a large fungous tumor. Upon the right side a similar large mass was observed. The largest tumors were found in the groins. They were of the same character as those already described, raw, bright red, fungous and bleeding at the slightest pressure. Upon the buttocks, thighs, calves, legs and feet elevated patches and excrescences were abundant. On account of the excessive itching the surface of the lesions were constantly torn, irritated and bleeding.

The patient lived at a distance, but visited me at stated intervals for perhaps eight months. During epithelial elements, each extending through the entire depth of the section of the specimen, dividing in some portions into epithelial columns with occasionally small interspaces. At and near the surface the epitheliai cells are large and somewhat decreasing in size from surface or margin as we descend into the body of the tumor, forming irregular cell columns and col. lections or nest-like arrangements."

The foregoing account certainly depicts an unusual condition of the skin. The existence for several years of prominent lesions of so extensive and well nigh universal distribution, with such slow decline of the general health, is not in consonance with our experi-

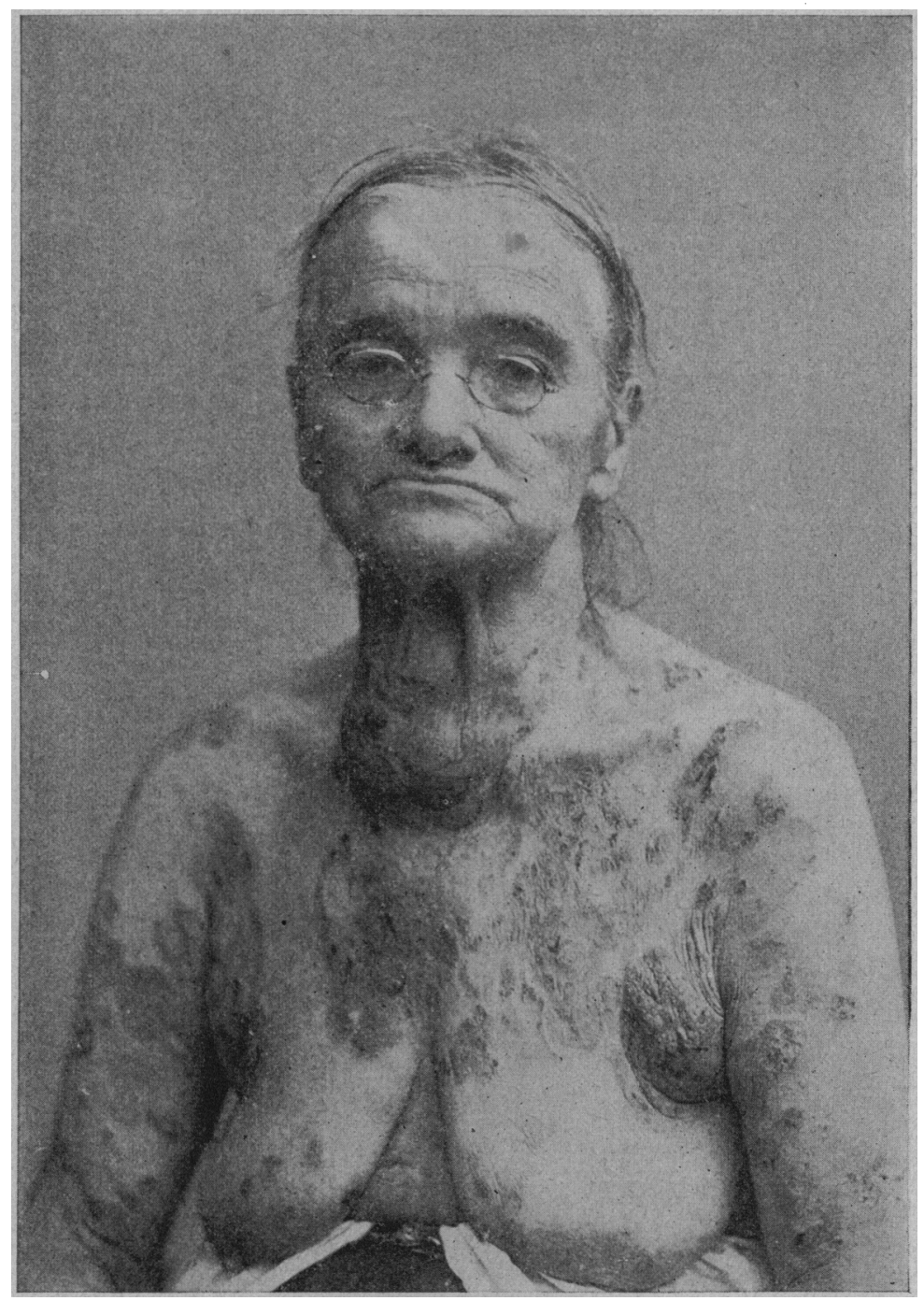

Mycosis Funroides (from Nature).

this period the disease seemed at times to improve, ence of malignant disease or severe infectious probut relapse invariably followed. The general health cesses. The microscopic examination was suggestive slowly declined and the patient succumbed in the neither of syphilis nor tuberculosis. The abundance summer of 1896 , during my absence from home. and arrangement of epithelial cells and the presence

The report of a microscopic examination of pieces taken during life from the tumors, reads as follows: "Scrapings of the surface showed single epithelial th cells of the squamous variety, together with some column-like cells with indistinct concentric rings or layers. Section revealed that the tumor was made up for the most part of epithelial cells with a vascular connective tissue, stroma or framework supporting the of a fibrous stroma points rather to carcinoma, but we can not admit the possibility of so many cancerous tumors existing for several years upon the surface without causing visceral involvement and cachexia. If, therefore, we are unable to place implicit dependence upon the revelations of the microscope, it only remains for us, relying upon the clinical manifestations and natural history of the oase, to pass in review 
those diseases capable of producing large and numerous fungous growths.

1. Syphilis. - The lesions undoubtedly bore no resemblance to the ordinary manifestations of syphilis. Nevertheless, the latter disease does sometimes give rise to fungous growths. Moist papules or mucous patches may sprout luxuriantly, but they do not cover the entire surface. The ulcerated tubercular syphilide may also assume the appearance of a vegetating growth, but this transformation is limited in locality and not so widely generalized as in the case which I have described. Furthermore, there was neither a history nor preceding evidence of syphilis. In doubtful cases we are accustomed to rely with some confidence upon the touch-stone of treatment. In this case anti-syphilitic treatment revealed nothing; it caused not the sligh test improvement.

2. Frambesia.-Syphilitic excrescences sometimes assume a berry-like form to which the name "frambesioid" syphilide has been applied by some writers. The disease, whose lesion is distinctively likened to a raspberry, is frambesia, or yaws, endemic in certain tropical countries, and occasionally seen in our extreme southern States. My patient had never lived farther south than Pennsylvania. The lesions of yaws are not so widely distributed, do not itch, frequently cause deep ulceration and unsightly cicatrices, is contagious, and is generally spread by sexual intercourse.

3. Tuberculosis.-Like syphilis, tuberculosis is the cause of obscure and intractable cutaneous affections. These, however, usually assume the forms of an infiltration tumor, followed by ulceration. If we accept lupus as the type of a mitigated tuberculosis of the skin, we can trace no resemblance either in the history or course of lupus with the case of which I now speak. It is true that papillary outgrowths sometimes occur in connection with lupous lesions, but these proliferations in themselves point to no individual disease. They develop whenever there is morbid hypernutrition of the papillary layer of the integ. ument. There is a form of tuberculosis accompanied by warty growths, known as tuberculosis verrucosa, but it is exceedingly rare, generally attacks the backs of the hands and occurs in those whose duties require them to handle cattle.

4. Pemphigus.-A variety termed pemphigus vegetans exhibits papillary excrescences, but these develop upon the basis of bullæ and this form of disease likewise is extremely rare. Here we have had no bullæ.

5. Sarcoma.-The tumors of this case certainly bore a considerable resemblance to sarcoma. As a rule, sarcoma advances rapidly, does not cause itching, and seldom manifests any tendency to spontaneous cure. There is, however, a generalized form of this disease, usually developing at or after middle age, beginning as hard edema, upon which nodules subsequently arise and grow slowly; these ultimately become tumors, which are at first hard and smooth but ultimately may ulcerate and assume a fungous aspect. These lesions may progress or retrocede and some may undergo spontaneous cure. The disease may finally involve mucous membranes and cause death. In other cases generalized sarcoma may begin with a solitary growth at some point, the single lesion being followed by other tumors. In this variety of disease the skin prior to ulceration is generally of a livid, brownish or brownish-red color. The tumors of the skin may also coexist with lesions of internal organs or lymphatic glands.
6. Mycosis fungoides.-There remains, finally, another rare affection, characterized by fungous tumors and corresponding more closely in lesions and symptoms to the present case than any of those hitherto mentioned. This is the disease known as mycosis fungoides. A synonymous title by which it has been designated, viz., multiple sarcomata, significantly points to the resemblances between the two maladies. Nevertheless, certain clinical distinctions are definitely marked. Mycosis fungoides does not always involve the lymphatic glands, and it never attacks internal organs. It pursues a tardy course, may last for many years and death is at last due to exhaustion, complication or intercurrent disease. Individual patches or tumors may subside without leaving any trace.

Mycosis fungoides usually begins during or after middle age. In a number of cases it has been preceded by some other cutaneons affection, as erythema, erysipelas, eczema, urticaria or furunculosis. The first phase of its development is by no means significant of its nature. Recurrent attacks of erythema, eczema or dermatitis occur for a long time; it may be for years. In the beginning these attacks are amenable to treatment, but with the lapse of time they become more and more intractable. Throughout the course of the disease itching is a prominent characteristic. In accordance with its clinical history mycosis fungoides has been divided into three periods or stages, the eczematiform, the lichenoid and the neoplastic. They all pursue a very tedious course. The patches of seeming eczema are of a dark or light red color. As the case progresses they become more or less raised above the surrounding surface and there may be a little scaling. In the commencement only the superficial layers of the skin are affected, but the deeper parts are at length involved. The affected surface gradually grows rougher and assumes an aspect which bears some resemblance to lichen planus.

Finally, the most distinctive stage, that of tumor formation, arrives. One or more of the lichenoid patches takes on increased development, and bulges forth as a veritable tumor of irregular or hemispheric form, somewhat firm consistence, bright red color and, as a rule, smooth surface. At this stage the tumors were compared by Alibert, who first described the disease, to ripe tomatoes. Subsequently the surface becomes excoriated or ulcerated, rough and fungous. Even at this advanced stage of development fungous tumors may be absorbed and disappear, leaving no race.

The division into stages is a broad and general one; the scheme of development is marked by many deviations. Lichenoid patches may, as in my case, coexist with numerous and large tumors; the three phases of the disease may be present upon the surface at the same time; the tumors may originate without any preceding manifestation. In a case reported, sheetlike infiltration was observed upon the base of the tongue, palate and in the larynx.

The duration of each stage is indefinite and the entire course of the disease is notably slow. It continues for several or many years. Sooner or later the patient begins to fail in general health. Death at length occurs either from gradual exhaustion, uncontrollable diarrhea or some other complication.

The actual cause of mycosis fungoides is still unknown. It is generally supposed to be of parasitic 
origin, but no constant organism has yet been demonstrated.

The treatment has not been very satisfactory. Arsenic, mercury, iodids and other remedies have been employed with variable degrees of success. At the present time we are only able to mitigate the symptoms or lesions of the disease without, probably, exerting much influence upon its course. External applications should be of a sedative and anti-pruritic character.

DISCUSSION.

Dr. Duhring-Nine cases out or ten are fatal. The diag nosis of the early stage is often very difficult even to an expert. The disease is more common than is generally supposed. The early lesions simulate other diseases very closely, and particularly the erythematous form of eczema. I recall one case which was treated for eczema and within two years the patient died. So it is of great importance to be able to diagnose a case before the fungoid condition makes its appearance, after which it is fatal. I remember a case which was almost clini cally like Dr. Shoemaker's, with the exception of the face lesions. The patient was treated with thyroid extract for three or four weeks and the lesions had largely disappeared. The family physician said that the patient had been able to follow his occupation (carpenter) up to two months ago and the main tumors had almost all disappeared. Then the patient suddenly became worse, the tumors increased in size, especially on the neck, axilla and chest, and within one month he sank very rapidly. There was extreme languor and the patient would not leave the bed, after which death occurred in two or three days. There is no hesitation in classing this affection as a distinct disease. I have seen two cases of sarcoma which were generalized and there was no suspicion of their being mycosis fungoidos. Both cases died within three months.

Dr. GotTHEIL - I had a very similar case and all forms of treatment were useless. The pruritus was so obstinate that the patient almost became insane. There were pinkish elevated plaques which itched very much and spread all over the body. The characteristic tumors appeared later. Numbers of previous cases had been diagnosed as eczema, psoriasis, tumors, etc. Every case of an intractable itching cutaneous disease should be suspected as a mycosis fungoides.

Dr. ALLEN-More attention ought to be paid to the premy. cotic stage. Sometimes the earlier leaions would be like a papular leprosy.

Dr. GILCHRIST-Coley's fluid, which is used in inoperable sarcomata, was tried on a case of mycosis fungoides, fungoid stage (a colored woman), in the Johns Hopkins Hospital, but although some temporary reduction in the size of the tumors had followed, yet no permanent good effects had resulted. I have examined a number of sections taken from three cases of mycosis fungoides and would not diagnose them as sarcomata as some observers have done.

\section{ULERYTHEMA SYCOSIFORME (LUPOID SYCOSIS) WITH REPORT OF TWO CASES.}

fresented to the Section on Cutaneous Medicine and Surgery, at the Forty-eighth Annual Meeting of the American Medical Association, held at Philadelphia, Pa.. June 1-4, 1897.

BY J. ABBOTT CANTRELL, M.D.

Professor of Diseases of the Skin in the Philadelphia Polyclinic and College for Graduates in Medicine, Dermatologist to the Philadelphia Hospital and to the Southern Dispensary.

AND JAY F. SCHAMBERG, M.D.

I bstructor on Diseases of the Skin in the Philadelphia Polyclinic and College for Graduates in Medicine, Dermatologist to the Union Mission Hospital. PHILADELPHIA, PA.

The following report is deemed worthy of presentation on occount of the rarity of the disease and the obscurity that has until recently surrounded it. About 1865 the disease was described by Milton under the name "lupoid sycosis." This author says "I have seen in some rare cases (of sycosis) a slow superficial form of ulceration beginning with minute tubercles and attacking the hair follicles causing fall- ing of the hair and slight though indelible cicatrices, a disorder which seems to me to be nothing more than lupus non-exedens." Unna, in 1889, described a disease which le terms ulerythema sykosiforme and reports a case in considerable detail. By ulerythema the latter writer means a pathologic process which goes on to the formation of atrophic scars without intermediate suppuration or ulceration; in other words, through the resorption of the cellular infiltrate. We append a full record of the case reported by Unua in order to show the similarity to the case which we describe.

Unna (Monatshefte f. Prak. Derm. 1889, ix, 134), records the case of a man 40 years of age, in whom the disease began in $\mathbf{1 8 7 7}$ as a small pustule upon the left cheek, which soon developed into a suppurating furuncle. A roundish, reddened, slightly elevated patch about the size of a German thaler gradually formed. This wept and was covered with an accumulation of crusts. The next year there appeared upon the hairy border of the left temple a similar patch covered with crusts. The weeping and crusts continued and the process gradually involved the whole of the left cheek. The hairs of the affected part became loose and could be easily lifted out. Upon extraction whitish nodules were visible upon the hair roots. After the disappearance of the hairs the affected area became pale and there developed smooth flat scar tissue. Two years later a patch developed in the beard of the right side while upon the left the process came to a standstill. Later the patch upon the left side spread over the whole cheek, thence down to the neck At the same time the moustache became involved upon both sides. Upon the reddened and slightly swollen skin there appeared crops of white vesicles with thick fluid whitish contents. These dried, were scratched open and formed branny crusts which re. sembled a starch-like dust and the face looked as if it had been powdered. From the beginning the patient shaved to prevent spreading of the affection, but now he rather believes that new points were thereby inoculated.

Present condition.-The patient looks robust and has a good healthy color. The hair of the scalp is blond and thick. The beard, which is likewise blond, is only present on the chin and neck. Both cheeks are bald except a small border on the rami of the jaws and there is evident extensive scarring. From a distance even these areas can be distinguished from the normal skin by a bluish red tint, an abnormal smoothness, and an almost satin-like shimmer. On closer examination numerous fine whitish fibers of cicatricial tissue are seen traversing the bluish red back. ground in a sieve-like manner. On the right side of the face the borders of the patch are quite elevated. Here are seen scattered brownish scales penetrated by hairs.

In the middle of the left upper lip there is a patch one-half to one centimeter in diameter in which there is total loss of hair. There is here smooth superficial scarring.

From the middle of the chin a reddish line of small firm papules descends toward the neck and thence turns to the right. On the left side of this line the skin is diffusely reddened and is covered with small round white scales. Near here is a group of five mustard seed sized exfoliation vesicles, three of which are pierced by hairs while the other two are not, but correspond however to follicular openings. 